\title{
Encephaloduroarteriosynangiosis for adult intracranial arterial steno-occlusive disease: long-term single-center experience with 107 operations
}

\author{
Nestor R. Gonzalez, MD, ${ }^{1,2}$ Joshua R. Dusick, MD, ${ }^{1}$ Mark Connolly, BS, ${ }^{1}$ Firas Bounni, MD, ${ }^{1}$ \\ Neil A. Martin, MD, ${ }^{1}$ Barbara Van de Wiele, MD, ${ }^{3}$ David S. Liebeskind, MD, ${ }^{4}$ and \\ Jeffrey L. Saver, MD ${ }^{4}$ \\ Departments of ${ }^{1}$ Neurosurgery, ${ }^{2}$ Radiology, ${ }^{3}$ Anesthesia, and ${ }^{4}$ Neurology, David Geffen School of Medicine, University of \\ California Los Angeles (UCLA), Los Angeles, California
}

\begin{abstract}
OBJECT Encephaloduroarteriosynangiosis (EDAS) is a form of revascularization that has shown promising early results in the treatment of adult patients with moyamoya disease (MMD) and more recently in patients with intracranial atherosclerotic steno-occlusive disease (ICASD). Herein the authors present the long-term results of a single-center experience with EDAS for adult MMD and ICASD.

METHODS Patients with ischemic symptoms despite intensive medical therapy were considered for EDAS. All patients undergoing EDAS were included. Clinical data, including recurrence of transient ischemic attack (TIA) and/or stroke, functional status, and death, were collected from a retrospective data set and a prospective cohort. Perren revascularization and American Society of Interventional and Therapeutic Neuroradiology/Society of Interventional Radiology (ASITN/SIR) collateral grades were recorded from angiograms.

RESULTS A total of 107 EDAS procedures were performed in 82 adults ( 36 with ICASD and 46 with MMD). During a median follow-up of 22 months, 2 (2.4\%) patients had strokes; both patients were in the ICASD group. TIA-free survival and stroke-free survival analyses were performed using the product limit estimator (Kaplan-Meier) method. The probability of stroke-free survival at 2 years in the ICASD group was $94.3 \%$ (95\% Cl 80\%-98.6\%). No patient in the MMD group suffered a stroke. The probability of TIA-free survival at 2 years was $89.4 \%(95 \% \mathrm{CI} 74.7 \%-96 \%)$ in ICASD and $99.7 \%$ (95\% Cl 87.5\%-99.9\%) in MMD. There were no hemorrhages or stroke-related deaths. Angiograms in $85.7 \%$ of ICASD and $92 \%$ of MMD patients demonstrated Perren Grade 3 and improvement in ASITN/SIR grade in all cases.
\end{abstract}

CONCLUSIONS EDAS is well tolerated in adults with MMD and ICASD and improves collateral circulation to territories at risk. The rates of stroke after EDAS are lower than those reported with other treatments, including intensive medical therapy in patients with ICASD.

http://thejns.org/doi/abs/10.3171/2014.10.JNS141426

KEY WORDS EDAS; encephaloduroarteriosynangiosis; atherosclerosis; moyamoya disease; intracranial arterial stenosis; stroke; vascular disorders

$\mathrm{E}$ NCEPHALODUROARTERIOSYNANGIOSIS (EDAS) is a form of indirect revascularization that has been used for the treatment of pediatric moyamoya disease (MMD) since the 1970s..$^{20}$ The application of EDAS for adults with MMD has shown promising results in the early postoperative period ${ }^{6}$ and its use has been extended to the treatment of select patients with intracranial atherosclerot- ic steno-occlusive disease (ICASD). ${ }^{7}$ ICASD is the most common cause of stroke worldwide. ${ }^{4,10,25}$ It accounts for at least $10 \%$ of all strokes in the United States ${ }^{21}$ and as much as 33\%-67\% of stroke in countries with predominantly Asian, Hispanic, and black populations. ${ }^{10}$ ICASD carries a worse prognosis than other stroke etiologies, with an annual rate of recurrent stroke and death of $15 \%$ despite

ABBREVIATIONS ASITN/SIR = American Society of Interventional and Therapeutic Neuroradiology/Society of Interventional Radiology; EDAS = encephaloduroarteriosynangiosis; ICA = internal carotid artery; ICASD = intracranial atherosclerotic steno-occlusive disease; IQR = interquartile range; MCA = middle cerebral artery; MMA = middle meningeal artery; MMD = moyamoya disease; SAMMPRIS = Stenting and Aggressive Medical Management for Preventing Recurrent stroke in Intracranial Stenosis; STA = superficial temporal artery; TIA = transient ischemic attack.

SUBMITTED June 24, 2014. ACCEPTED October 16, 2014.

INCLUDE WHEN CITING Published online June 12, 2015; DOI: 10.3171/2014.10.JNS141426.

DISCLOSURE Research reported in this publication was supported by the National Institute of Neurological Disorders and Stroke of the National Institutes of Health under Award Number K23NS079477. The content is solely the responsibility of the authors and does not necessarily represent the official views of the National Institutes of Health. 
intensive medical management, ${ }^{3}$ and as high as $25 \%$ in certain populations. ${ }^{23,24}$ Interventions such as angioplasty and stenting or bypass surgery have failed to improve outcomes in ICASD ${ }^{1,2,19}$ and have not been tested in pivotal clinical trials for MMD. Although the short-term (30-day) stroke and death rates with angioplasty alone have been shown to be low (3.3\%) in one study subgroup, the longer-term (2-year) outcomes of these techniques have been disappointing (18\% 2-year stroke rate in the SAMMPRIS [Stenting and Aggressive Medical Management for Preventing Recurrent stroke in Intracranial Stenosis] trial) and no better than medical management alone.,, 22 Demonstrating the long-term effectiveness of the EDAS procedure in generating neovascularization and preventing transient ischemic attacks (TIAs) or stroke in patients with ICASD or MMD is a key step in establishing this technique as a useful alternative for their treatment. Herein, we present the long-term clinical and midterm angiographic results of our center's utilization of EDAS surgery in adult patients with ICASD and MMD, based on the application of a meticulous protocol of operative and perioperative management for these conditions.

\section{Methods}

All adult patients with a diagnosis of ICASD or MMD treated with EDAS surgery at our institution from October 1989 to February 2014 were included. This population comprises a retrospectively collected series of the initial cases between 1989 and 2007 and a prospectively monitored cohort of the surgeries since then. Our institutional review board approved this study.

\section{Criteria for Selecting Patients for EDAS Treatment}

Patients with symptomatic intracranial arterial stenosis, either due to MMD or ICASD, are considered for indirect revascularization by EDAS if they have had recent (within 30 days) TIAs or nondisabling stroke in the territory of the stenosis, despite optimized medical management with antiplatelet medications in cases of MMD and with antiplatelet medications, statins, adequate blood pressure control, and secondary risk factor management (diabetes control, smoking cessation, weight loss, and exercise) in cases of ICASD. Initial workup includes MRI with perfusion imaging as well as catheter cerebral angiography. The earliest patients from this series did not undergo perfusion MRI as it was not available at that time. Patients with evidence of hypoperfusion and poor collateral flow (American Society of Interventional and Therapeutic Neuroradiology Society of Interventional Radiology [ASITN/SIR] Grades 0-2) with persistent ischemic symptoms are considered as candidates for surgical treatment with EDAS. ${ }^{12}$ Patients with atherosclerotic disease undergo a standardized cardiac evaluation prior to surgery.

A diagnosis of highly probable MMD is made in individuals who present with evidence of bilateral intracranial disease involving predominantly the internal carotid arteries and/or proximal anterior and middle cerebral arteries with no evidence of vessel calcification and with angiographic collaterals characteristic of MMD. Indi- viduals with unilateral intracranial internal carotid artery (ICA) disease without calcification of the arterial wall, without risk factors for atherosclerosis, and with angiographic collaterals characteristic of MMD are considered probable cases of MMD and were included as MMD cases. A diagnosis of ICASD is made in individuals with intracranial arterial disease with evidence of vessel wall calcification and history of atherosclerotic risk factors, including a history of hypertension, dyslipidemia, diabetes mellitus, smoking, and/or coronary or peripheral vascular atherosclerotic disease. Details of the diagnostic criteria are provided in the Supplementary Materials.

\section{Perioperative and Anesthesia Care}

Optimal care of adult patients with intracranial arterial steno-occlusive disease requires careful preoperative preparation, intraoperative anesthesia management, and postoperative critical care. Throughout the complete process, the goal is to avoid any hypotension, hypovolemia, anemia, or vasoconstriction, while maintaining antiplatelet medications and controlling other stroke risk factors. To achieve this goal we have developed strict guidelines for management of these patients, including anesthesia management and postoperative critical care. All patients undergoing surgery receive a dose of $325 \mathrm{mg}$ aspirin on the date of surgery and for at least 3 days prior to the procedure. The baseline systolic blood pressure at which the patient is asymptomatic is identified in clinic prior to surgery and is considered to be the minimum systolic blood pressure limit for surgical anesthesia as well as postoperative care. The maximum systolic blood pressure goal is set at $200 \mathrm{~mm} \mathrm{Hg}$. Hyperventilation is avoided during surgery to reduce the risk of vasoconstriction and hypoperfusion. After intubation, the end-tidal $\mathrm{CO}_{2}$ is maintained in the range of $35-45 \mathrm{~mm} \mathrm{Hg}$. An antiseizure medication loading dose is administered over 60 minutes to avoid hypotension. Mannitol and corticosteroids are not administered. The hematocrit is monitored and maintained in the $30 \%-50 \%$ range. All patients receive an arterial catheter for blood pressure monitoring as well as a central venous catheter for monitoring central venous pressure. The goal of fluid management is to maintain euvolemia to modest hypervolemia. Body temperature is monitored, and the goal is normothermia. Systemic hypothermia and/or barbiturates are not used, as there is no temporary occlusion of intracranial arteries during EDAS.

\section{Surgical Technique}

The surgical technique for EDAS has been published before. ${ }^{6}$ However, performing this operation in adults, in particular in those with ICASD, requires specific technical modifications. The most relevant modifications include maintenance of antiplatelet medications during the surgery, microscopic dissection of the donor arteries (superficial temporal artery [STA] and middle meningeal artery [MMA]), minimization of cauterization of donor vessel branches, dissection of the layers of the dura mater, and extensive microscopic arachnoid dissection. A detailed description of the surgical procedure is provided in the Supplementary Materials for this paper. 


\section{Postoperative Management}

Following surgery, patients are monitored in recovery and in the neurocritical care unit with hourly neurological examinations and vital sign monitoring. Nursing staff has been trained to perform National Institutes of Health Stroke Scale evaluations and to recognize and report subtle changes in neurological examination findings. Strict blood pressure and fluid management goals are monitored and maintained as during anesthesia. Only drips of nicardipine and norepinephrine are used as needed to maintain blood pressure within the goal range, avoiding as-needed medications. Daily aspirin is continued postoperatively ( $325 \mathrm{mg}$ by mouth daily or $300 \mathrm{mg}$ by rectum daily). Antiseizure medication is continued for 7 days. Patients are transferred to the floor when they are in stable neurological condition and do not require drips to maintain their blood pressure in the asymptomatic range. The patients are discharged home once their blood pressure is stable without as-needed medications and when they have been cleared by physical and occupational therapy. Patients whose data are being collected prospectively have their neurological preoperative, immediate postoperative, and return visit assessments completed by neurologists from the stroke center, and their evaluations are used in this study. In cases with retrospective data collection, the evaluation from independent neurologists from the stroke center was also preferred for the functional evaluations used for this study.

Clinical data, including general demographic data, surgery date, last follow-up date, neurological condition at baseline and last follow-up, presence of recurrent TIA and/or stroke in the revascularized territory, and strokerelated death, were collected from all patients' medical records and case report forms. Additionally, the last followup cerebral angiography date was recorded. The Perren revascularization grade, ${ }^{18}$ ASITN/SIR collaterals grade,,$^{12}$ and presence of direct filling of intracranial vessels by selective external carotid artery injection were recorded for those patients with suitable postoperative angiograms for evaluation of these scales.

\section{Statistical Analysis}

Demographics and outcomes are reported as the mean \pm SD for normally distributed variables and median and interquartile range (IQR) for skewed variables. Comparisons between the MMD and ICASD groups were made with Student t-tests for normally distributed variables. Wilcoxon rank-sum tests were used for nonparametric comparison between nonnormally distributed variables, and the chi-square test was used for comparison of proportions. TIA-free survival and stroke-free survival analyses were performed using the product limit estimator (Kaplan-Meier) method; an administrative limit censoring time of 72 months was selected. Survival comparisons between groups were done using the log-rank (Mantel) test. The $\log (-\log (\operatorname{Surv}))$ plot versus time showed an approximate linear relation, indicating that the Weibull parametric distribution should provide a good fit for the data. Using the Weibull parametric fit the survival probabilities at 12,24 , and 36 months were calculated. All statistical analyses were performed with JMP Pro 11 (SAS Institute Inc.).

\section{Results}

\section{Patient Demographics}

We performed EDAS surgeries on 107 hemispheres in 82 adult patients, 36 with ICASD and 46 with MMD, with a median follow-up of 24 months for patients with ICASD and 20 months for patients with MMD. General descriptive statistics, comparing groups, are presented in Table 1. There were no statistical differences in sex distribution or duration of clinical follow-up between the 2 groups. However, there is an expected difference in age between the ICASD and MMD patients (mean $50.4 \pm 16.23$ years vs $36.5 \pm 9.19$ years, respectively; $p<0.0001$ ). The majority of patients in both groups were Caucasian $(50 \%$ in the ICASD and 58.7\% in the MMD group). There were no significant differences in race or ethnicity between groups. Angiographic follow-up was longer for MMD patients (median 19 months vs 7 months for ICASD cases, $p$ $=0.04)$.

\section{Vascular Lesions}

In the MMD group, bilateral disease was present in 26 patients $(56.5 \%)$ and unilateral disease in 20 patients (43.5\%). In the ICASD group, bilateral disease was present in 4 patients $(11.1 \%)$ and unilateral disease in 32 patients $(88.9 \%)$. None of the patients with bilateral disease had critical stenosis $(>70 \%)$ on the contralateral side. In the ICASD group, the critical lesion on the operated side involved the intracranial ICA in 17 patients $(47 \%)$, the $\mathrm{M}_{1}$ segment of the middle cerebral artery (MCA) in 12 cases (33\%), the ICA and $\mathrm{M}_{1}$ segment in 4 patients (11\%), the $\mathrm{M}_{2}$ MCA in 2 patients $(6 \%)$, and the MCA and anterior cerebral artery in 1 patients. The degree of stenosis in the patients with ICASD ranged from $70 \%$ to complete occlusion, with a median stenosis of 99\% (IQR 95\%-100\%). There were complete occlusions in 12 (33.3\%) of the patients.

\section{Clinical Outcomes}

During the immediate operative period and within 30 days following EDAS surgery, there was 1 ischemic stroke in the revascularized territory in a patient in the ICASD group ( $0.9 \%$ of operations [1 of 107]) and no stroke-related deaths or hemorrhagic complications following the 107 operations. The ischemic stroke occurred in a 53-year-old man with end-stage kidney disease who suffered worsening of aphasia, with MRI findings confirming a new area of ischemia in the left frontal lobe. The patient had partial improvement of symptoms at his 1-month follow-up. During the full follow-up period 10 patients (12.2\%) experienced new TIAs referable to the revascularized territory. There was no significant difference in the proportion of patients with TIAs between the 2 groups, although they were nominally more common in patients with ICASD (MMD: $8.7 \%$ vs ICASD: $16.7 \% ; p=0.28$ ). The duration of TIA-free survival was excellent for both groups, with no statistically significant difference, as shown in the Kaplan Meier curves in Fig. 1. The calculated probability of TIAfree survival at 2 years, at which point $53 \%$ of ICASD cases and $49 \%$ of MMD cases were still being followed, was $89.4 \%$ (95\% CI $74.7 \%-96 \%$ ) for ICASD and $99.7 \%(95 \%$ 
TABLE 1. Demographic data and follow-up information

\begin{tabular}{|c|c|c|c|c|}
\hline Variable & Total $(\mathrm{n}=82)$ & $\operatorname{MMD}(n=46)$ & ICASD $(n=36)$ & p Value \\
\hline Mean age in yrs $( \pm S D)$ & $42.6 \pm 14.4$ & $36.5 \pm 9.2$ & $50.4 \pm 16.3$ & $<0.0001^{*}$ \\
\hline Female sex & $75.6 \%$ & $78.3 \%$ & $72.2 \%$ & \\
\hline Ethnicity (non-Hispanic)† & $82.9 \%$ & $80.4 \%$ & $86.1 \%$ & \\
\hline \multicolumn{5}{|l|}{ Race } \\
\hline White & $54.9 \%$ & $58.7 \%$ & $50.0 \%$ & \\
\hline Asian & $34.2 \%$ & $36.9 \%$ & $30.6 \%$ & \\
\hline Black & $8.5 \%$ & $4.4 \%$ & $13.9 \%$ & \\
\hline Other & $2.4 \%$ & 0 & $5.5 \%$ & \\
\hline Median clinical FU in mos (IQR) & $22(6.5-47.5)$ & $20(5-44.5)$ & $24(7-49.75)$ & \\
\hline Median angiographic FU in mos (IQR) & $8(6-28.75)$ & $19(7-30.5)$ & $7(6-16.25)$ & $0.04 \dagger$ \\
\hline \multicolumn{5}{|c|}{$\begin{array}{l}\text { FU = follow-up. } \\
\text { * This } p \text { value was calculated using the Student t-test. } \\
\dagger \text { Chi-square calculations for the differences between race groups and Fisher's exact test for the comparison between Hispanic and non- } \\
\text { Hispanic showed no significant differences. } \\
\ddagger \text { This } p \text { value was calculated using the Wilcoxon rank-sum test. }\end{array}$} \\
\hline
\end{tabular}

CI 87.5\%-99.9\%) for MMD. Table 2 provides the annual probability of TIA-free survival.

During the full follow-up period 2 patients $(2.4 \%$ [2/82]) experienced strokes in the revascularized territory, both ischemic. They occurred in the ICASD group (5.6\% [2/36]). These include the perioperative stroke described above and an ischemic stroke in an 83-year-old female, 3 months after surgery. This patient died of respiratory complications associated with aspiration pneumonia. This is the only stroke-related death in this cohort. The strokefree survival stabilized after the initial 3-month postoperative period as shown in the Kaplan-Meier curve in Fig. 1. The calculated probability of stroke-free survival at 2 years, at which point $53 \%$ of ICASD cases were still being followed, was $94.3 \%$ (95\% CI 80.0\%-98.6\%). Table 2 provides the annual probability of stroke-free survival in the ICASD group. There were no strokes in the MMD group. Patients' adherence to follow-up visits was $83.3 \%$ for ICASD patients and 52.2\% for MMD cases.

Functional outcome data demonstrated good outcomes (modified Rankin Scale [mRS] score of 0-2) in $81.7 \%$ globally. Patients with ICASD had mRS scores $\leq 2$ in $77.8 \%$ of cases before surgery and $83.3 \%$ of cases after EDAS. Patients with MMD had mRS scores $\leq 2$ in $82.6 \%$ of cases before surgery and $80.4 \%$ after EDAS surgery. Figure 2 shows the mRS score distribution at baseline and at last follow-up.

\section{Angiographic Outcomes}

Postoperative angiograms were available for review in 53 patients ( 28 patients with ICASD and 25 with MMD), at a median of 7 months (interquartile ratio [IQR] 6-15.5 months) after surgery in the ICASD group and 16 months (IQR 7.8-32.8 months) in the MMD group. ASITN/SIR grades could be determined in 43 patients ( 24 with ICASD and 19 with MMD). Overall, patients in both groups (85.7\% in the ICASD group and $92 \%$ in the MMD group) demonstrated excellent revascularization (Perren Grade 3) without a significant difference between the groups. There were no cases without evidence of revascularization (Perren Grade 1).

Collaterals, as indicated by ASITN/SIR grade, improved in all patients. There was no significant difference in the proportions of ASITN/SIR grades postoperatively between the 2 groups. Complete collaterals to the territory at risk (ASITN/SIR Grades 3 and 4) were seen in 62.5\% of ICASD patients and $52.6 \%$ of MMD patients. Table 3 describes the specific ASITN/SIR grades for each group.

\section{Early Angiographic Results}

A subset of 17 patients (3 with MMD and 14 with ICASD) had early angiograms, within 6 months after EDAS surgery. The early angiograms were performed at a median of 168 days (IQR 102-180 days; range 7-180 days) postoperatively. Fourteen patients had excellent revascularization (Perren Grade 3), 2 patients had moderate revascularization (Perren Grade 2), and 1 patient, whose angiogram was obtained 7 days after surgery, did not demonstrate visible collaterals (Perren Grade 1). This very early angiogram is of particular interest because, although no new vessels were observed, after selective contrast injections of the external carotid donor branches, there was evidence of transit of the contrast medium into the superior sagittal sinus. This finding was not present on the preoperative angiogram, suggesting the possibility of early small anastomoses of diameters below the resolution of digital subtraction angiograms of $200 \mu \mathrm{m}$ (Fig. 3). In addition, in this early angiogram group there were 2 patients with very early angiograms at 1 and 2 months after EDAS that already demonstrated revascularization (Perren Grades 3 and 2).

\section{Discussion}

The surgical induction of collateral vessels to supply vascular territories at risk for stroke in patients with stenoocclusive disease is an intriguing concept. On one hand, the process of neovascularization from arteries of the external carotid circulation, the formation of spontaneous connec- 

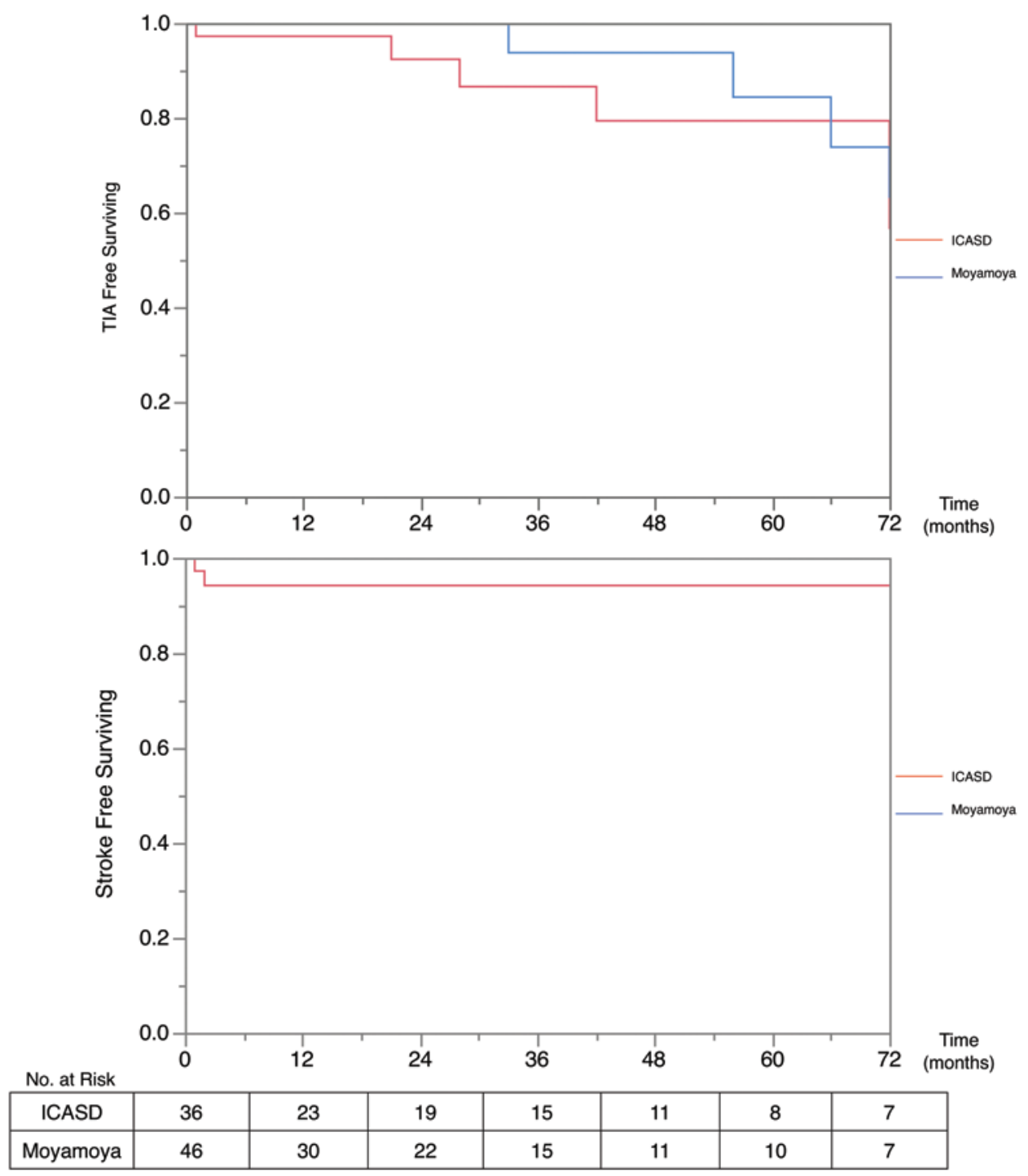

FIG. 1. TIA-free and stroke-free Kaplan-Meier survival curves for MMD and ICASD. Administrative censoring was applied at 72 months. Log-rank comparison demonstrated no significant differences between groups $(p=0.27)$. The moyamoya group had no strokes during follow-up.

tions to the intracranial vessels, and their maturation into vessels capable of supporting the cerebral circulation offer unprecedented opportunities to understand mechanisms of angiogenesis and arteriogenesis and their potential role as therapeutic tools. ${ }^{9}$ On the other hand, only if this process can protect individuals at risk from stroke early enough and in the long term with stable mature collaterals should EDAS be considered a viable alternative to other strategies of treatment. Although no pivotal clinical trials have been performed in the setting of adults with MMD, various forms of indirect revascularization, including EDAS, have been used in the treatment of MMD for decades. ${ }^{20} \mathrm{We}$ have previously shown that adult patients with MMD typically have good outcomes in the short term, with resolution of ischemic symptoms in most patients. ${ }^{6}$ Furthermore, follow-up angiograms in those early experiences demonstrated significant neovascularity, including enlargement of the feeding external carotid artery branches (STA and MMA), new vascular blush, and direct filling of intracranial arteries from selective external carotid artery injection in a majority of patients. The long-term clinical and midterm angiographic results presented here confirm the early findings of the utility of EDAS to protect against TIA, stroke, and stroke-related death, with excellent event-free survival rates and evidence of neovascularization with no hemorrhagic complications in adult patients with MMD.

The application of EDAS for patients with intracranial atherosclerosis was discouraged by the results of the small case series of Komotar et al., who studied 12 symptomatic patients. ${ }^{15}$ However, they did not apply indirect revascularization for intracranial stenosis, but rather for intracranial occlusion (11 of the 12 patients). Several aspects of their patient selection and management differ from our approach; they included patients with severe diffuse disease in multiple vascular territories, there was no consistent intensive medical management, strict intraoperative and postopera- 
TABLE 2. Annual probability of TIA-free and stroke-free survival

\begin{tabular}{ccccc}
\hline Time & $\begin{array}{c}\text { ICASD } \\
(\%)\end{array}$ & $95 \% \mathrm{Cl}$ & $\begin{array}{c}\text { MMD } \\
(\%)\end{array}$ & $95 \% \mathrm{Cl}$ \\
\hline TIA-free survival & & & & \\
\hline 1 & 95.5 & $83.3-98.9$ & 99.9 & $93.5-100$ \\
\hline 2 & 89.4 & $74.7-96.0$ & 99.7 & $87.5-99.9$ \\
\hline 3 & 83.0 & $65.9-92.4$ & 98.1 & $81.1-99.8$ \\
\hline Stroke-free survival & & & & \\
\hline 1 & 95.5 & $83.1-98.9$ & & \\
\hline 2 & 94.3 & $80.0-98.6$ & & \\
\hline 3 & 93.5 & $77.4-98.4$ & & \\
\hline
\end{tabular}

tive protocols for blood pressure and fluid management were lacking, and antiplatelet medications were not administered during surgery. These details of our approach and the technical modifications with scrupulous microsurgical vascular dissection and hemostasis are key elements in the revascularization of patients with ICASD. Patients with ICASD represent a more challenging group than those with MMD, as they are typically older, have higher cardiac risk, and have been shown to be more resistant to other forms of treatment, such as direct bypass and angioplasty and stenting, with high recurrent stroke rates reported in the literature despite intensive medical management. . $^{1-3,11,13,19,23}$

Observations in clinical trials have confirmed an important role for collateral circulation in averting stroke in ICASD, providing pathophysiological support for the hypothesis that collateral enhancement by EDAS could improve outcome. Evaluation of the WASID (Warfarin-
Aspirin for Symptomatic Intracranial Disease) population demonstrated that when the degree of arterial luminal stenosis was severe $(\geq 70 \%)$, the presence of good collaterals had a dramatic role in averting stroke. Presence of poor collaterals increased the risk of stroke by 6-fold in the compromised vascular territory (no collaterals or poor vs good, 30\% vs 5\%, HR 6.05 [95\% CI 1.41-25.92]; log rank, $\mathrm{p}=0.0056) .{ }^{17}$ Angiographic analysis of the collateral circulation of patients enrolled in SAMMPRIS found that none of 117 patients with good collaterals (ASITN/SIR Grades 3 and 4, 66 in the medical arm and 51 in the stent arm) had strokes within 30 days of enrollment. ${ }^{16}$ While some patients with stenosis develop adequate leptomeningeal collaterals on their own, those patients who remain symptomatic can potentially benefit from the additional collaterals created as a result of the EDAS procedure. We have previously reported on the initial group of ICASD patients managed with EDAS with good early results. ${ }^{7}$ Here we have expanded on that series with additional patients and longer follow-up demonstrating excellent overall control of ischemic symptoms and a very low rate of recurrent stroke after treatment, comparable to that obtained in patients with MMD, for whom the treatment has an established role.

While it has generally been assumed that EDAS takes many months to develop significant collaterals, Perren et al. demonstrated evidence of neovascularity much earlier in MMD patients..$^{18}$ Our patients with angiographic follow-up, including 14 patients with ICASD with early post-EDAS angiograms, also demonstrated good collateral formation as early as 1 month after surgery, with the suggestion of possible early formation of collaterals of a size below the resolution of angiography as early as 7 days

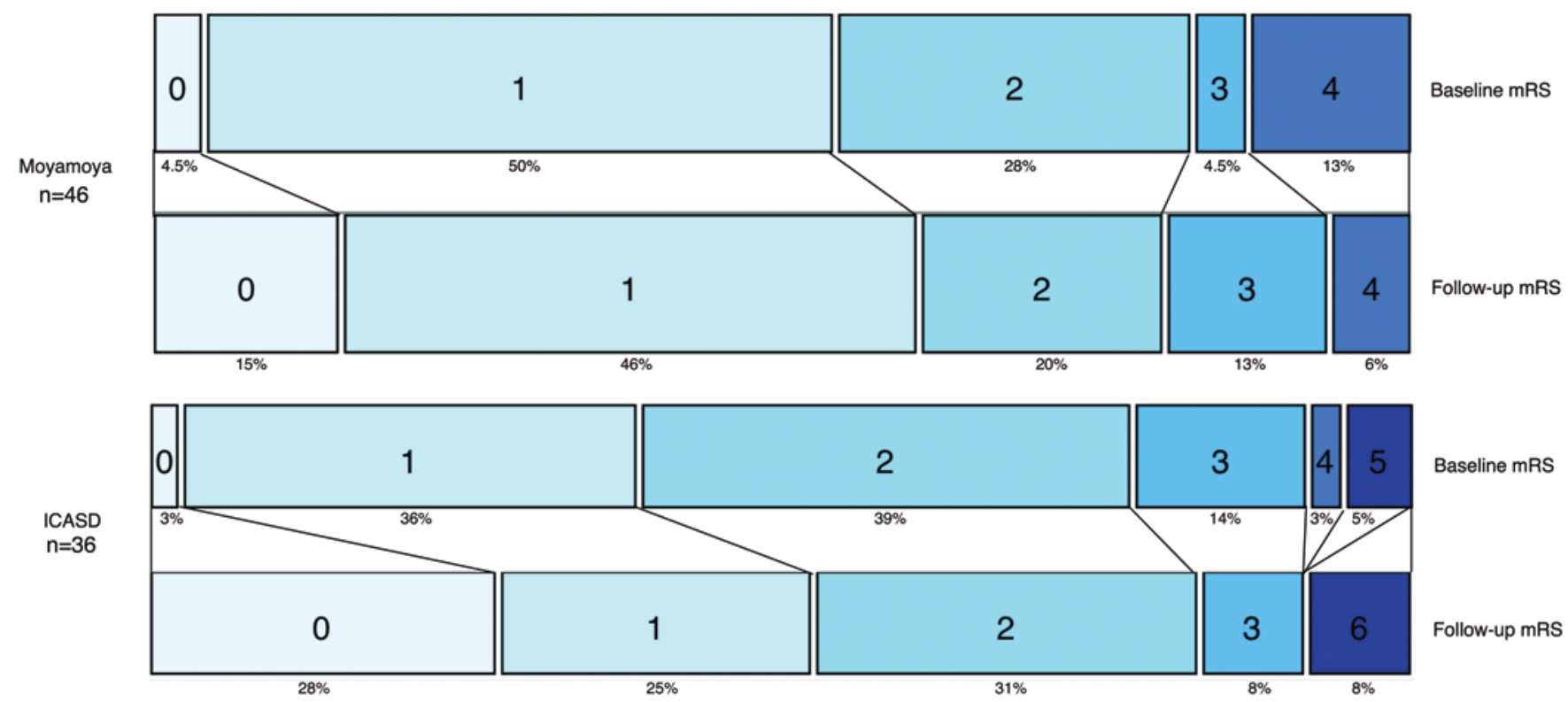

FIG. 2. Stacked bar chart depicting the distribution of the mRS score in patients with MMD and ICASD at baseline and last followup. In the ICASD group, $77.8 \%$ and $83.3 \%$ of patients had mRS scores $\leq 2$ before and after EDAS surgery, respectively. In the MMD group, patients $82.6 \%$ and $80.4 \%$ of patients had mRS scores $\leq 2$ before and $80.4 \%$ after EDAS surgery, respectively. Three patients in the ICASD group had an mRS score of 6: 1 stroke-related death and 2 additional deaths during the full cohort follow-up associated with cardiac issues. Those patients did not present with any strokes up to the time of death and had poor mRS scores at presentation ( 1 had an mRS score of 4 and 2 had an mRS score of 5). 
TABLE 3. ASITN grades on follow-up angiography

\begin{tabular}{cccc}
\hline \multirow{2}{*}{$\begin{array}{c}\text { ASITN/SIR } \\
\text { Grade }\end{array}$} & \multicolumn{3}{c}{ No. of Patients (\%) } \\
\cline { 2 - 4 } & ICASD & MMD & Total \\
\hline 1 & $3(12.5)$ & $0(0)$ & $3(7.0)$ \\
\hline 2 & $6(25)$ & $9(47.4)$ & $15(34.9)$ \\
\hline 3 & $2(8.3)$ & $3(15.8)$ & $5(11.6)$ \\
\hline 4 & $13(54.2)$ & $7(36.8)$ & $20(46.5)$ \\
\hline Total & 24 & 19 & 43 \\
\hline
\end{tabular}

* Percentages are based on the totals in each column.

after the operation. Although only a small subset of our series had these earlier angiograms for analysis, these early examples do suggest that revascularization after EDAS may occur in many patients far earlier than previously assumed.

The standardized operative and perioperative care of these patients described here plays a fundamental role in maximizing the chances of the procedure to induce revascularization while minimizing the perioperative risk for hypoperfusion, embolisms, and ischemic injury. While direct bypass creates a new source of flow to ischemic territories immediately, EDAS takes some time to develop new collateral circulation. The early sudden increase in flow induced by direct bypass can potentially produce hyperperfusion and hemorrhagic complications. ${ }^{8,14}$ In addition, in the EC-IC bypass trial, patients with intracranial stenotic lesions consistently fared worse following bypass because the sudden change in flow dynamics across the stenotic segment may produce stasis of flow and thrombosis, which can embolize and/or propagate to occlude lenticulostriate arteries. ${ }^{1,13}$ However, direct bypass has the potential advantage that it may provide immediate protection from ischemia. Our findings support the concept that strict medical management protects patients from ischemia in the initial period. The early angiographic evidence of vessel formation after EDAS and the clinical results we are reporting both in the immediate postoperative and longterm periods emphasize the safety and effectiveness of the procedure in both MMD and ICASD, with the presence of mature collaterals from the external circulation that were not associated with hemorrhagic complications.

Some surgical technical aspects that have become standard in our management include scrupulous microscopic dissection of both the STA and MMA. The MMA contributes a significant portion of the neovascularity seen after EDAS in most patients. In fact, we have previously reported that the percentage enlargement of the proximal portion of the MMA seen on postoperative angiograms, due to increased blood flow through the vessel, was $49 \%$ in MMD patients, very similar to the increase of $51 \% \mathrm{ob}-$ served in the STA. ${ }^{6}$

Comparison of patients being treated for MMD versus ICASD revealed no significant differences in either clinical outcome or angiographic revascularization. While the goal of this study was not to directly compare MMD and ICASD patients, given that the pathologies are different with different natural histories, our findings suggest that EDAS is an effective treatment strategy for both despite

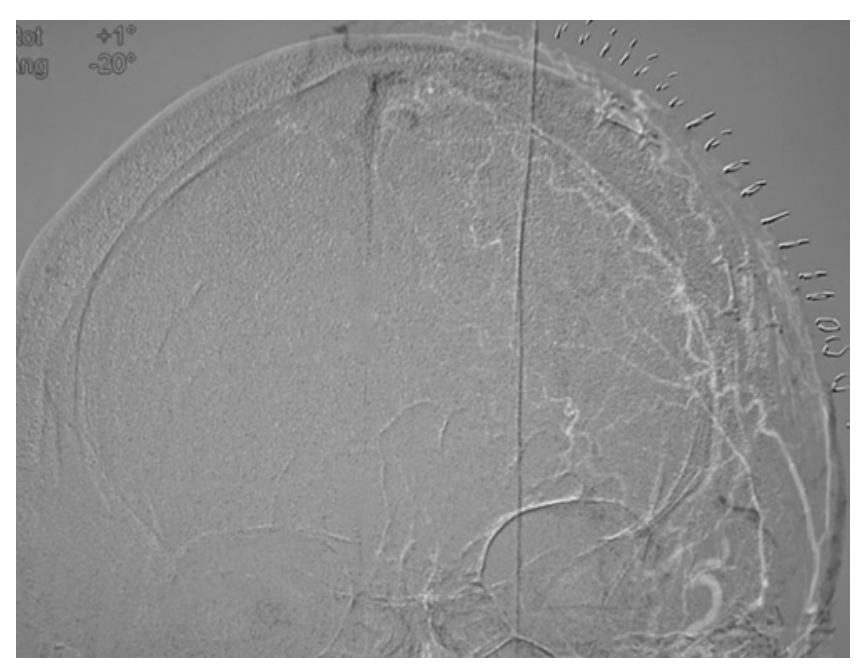

FIG. 3. Very early digital subtraction angiogram obtained 7 days after EDAS surgery in a patient with ICASD. Anteroposterior view with masking of the early arterial phase to confirm the absence of contamination from ICA contrast during the injection. There is no visualization of neovascularization; however, there is evidence of contrast medium transit into the superior sagittal sinus, not present on the preoperative angiograms, suggesting the presence of early collateral connections between the extra- and intracranial circulation with caliber below the resolution of DSA of $200 \mu \mathrm{m}$.

these differences. Our results show that both groups generally tolerate the procedure well and have high rates of TIA-free and stroke-free survival following surgery. The rate of stroke in our cohort of patients with ICASD at a median follow-up time of 24 months (5.6\%) is lower than the probability of stroke at 2 years in both the medical group $(18 \%)$ and angioplasty and stenting group $(21 \%)$ reported in the final results of SAMMPRIS. ${ }^{5}$ However, these findings require further corroboration and are currently being evaluated in a prospective Phase II clinical trial of EDAS for ICASD (NCT01819597, ClinicalTrials.gov), adhering strictly to the same inclusion criteria used in SAMMPRIS. If this trial is successful, an additional pivotal study will be required to confirm the results.

This study contains a portion of data collected retrospectively, which introduces some limitations. Several efforts have been made to address these limitations, including the report of all cases performed, a detailed description of the inclusion and diagnostic criteria, application of a homogeneous protocol of medical and surgical treatment, independent evaluations by stroke neurologists, and very good patient adherence to the follow-up schedule, in particular in the ICASD group.

\section{Conclusions}

Indirect revascularization by EDAS is a well-tolerated treatment that improves collateral circulation to the territory at risk. The application of strict operative and perioperative protocols reported here appears to reduce the rate of TIA and stroke in both the early and long-term period in patients with ICASD and MMD. EDAS is a useful alternative for the treatment of ICASD, and further evaluation in pivotal trials is warranted. 


\section{Acknowledgments}

We are grateful for the support of the UCLA Comprehensive Stroke Center faculty, fellows, and staff, the Department of Anesthesia's commitment to the care of our patients, and the dedication of the research coordination team led by Antoinette Anderson at the Gonzalez Cerebrovascular Laboratory at UCLA.

\section{References}

1. Awad I, Furlan AJ, Little JR: Changes in intracranial stenotic lesions after extracranial-intracranial bypass surgery. J Neurosurg 60:771-776, 1984

2. Chimowitz MI, Lynn MJ, Derdeyn CP, Turan TN, Fiorella D, Lane BF, et al: Stenting versus aggressive medical therapy for intracranial arterial stenosis. N Engl J Med 365:993-1003, 2011

3. Chimowitz MI, Lynn MJ, Howlett-Smith H, Stern BJ, Hertzberg VS, Frankel MR, et al: Comparison of warfarin and aspirin for symptomatic intracranial arterial stenosis. N Engl J Med 352:1305-1316, 2005

4. De Silva DA, Woon FP, Lee MP, Chen CP, Chang HM, Wong MC: South Asian patients with ischemic stroke: intracranial large arteries are the predominant site of disease. Stroke 38:2592-2594, 2007

5. Derdeyn CP, Chimowitz MI, Lynn MJ, Fiorella D, Turan TN, Janis LS, et al: Aggressive medical treatment with or without stenting in high-risk patients with intracranial artery stenosis (SAMMPRIS): the final results of a randomised trial. Lancet 383:333-341, 2014

6. Dusick JR, Gonzalez NR, Martin NA: Clinical and angiographic outcomes from indirect revascularization surgery for Moyamoya disease in adults and children: a review of 63 procedures. Neurosurgery 68:34-43, 2011

7. Dusick JR, Liebeskind DS, Saver JL, Martin NA, Gonzalez NR: Indirect revascularization for nonmoyamoya intracranial arterial stenoses: clinical and angiographic outcomes. J Neurosurg 117:94-102, 2012

8. Fujimura M, Shimizu H, Inoue T, Mugikura S, Saito A, Tominaga T: Significance of focal cerebral hyperperfusion as a cause of transient neurologic deterioration after extracranialintracranial bypass for moyamoya disease: comparative study with non-moyamoya patients using $\mathrm{N}$-isopropyl-p-[(123)I] iodoamphetamine single-photon emission computed tomography. Neurosurgery 68:957-964, discussion 964-965, 2011

9. Gonzalez NR, Liebeskind DS, Dusick JR, Mayor F, Saver J: Intracranial arterial stenoses: current viewpoints, novel approaches, and surgical perspectives. Neurosurg Rev 36:175185,2013

10. Gorelick PB, Wong KS, Bae HJ, Pandey DK: Large artery intracranial occlusive disease: a large worldwide burden but a relatively neglected frontier. Stroke 39:2396-2399, 2008

11. Group EIBS: The International Cooperative Study of Extracranial/Intracranial Arterial Anastomosis (EC/IC Bypass Study): methodology and entry characteristics. Stroke 16:397-406, 1985

12. Higashida R, Furlan A, Roberts H, Tomsick T, Connors B, Barr J, et al: Trial design and reporting standards for intraarterial cerebral thrombolysis for acute ischemic stroke. J Vasc Interv Radiol 14:S493-S494, 2003

13. The EC/IC Bypass Study Group: Failure of extracranialintracranial arterial bypass to reduce the risk of ischemic stroke. Results of an international randomized trial. N Engl J Med 313:1191-1200, 1985

14. Kim JE, Oh CW, Kwon OK, Park SQ, Kim SE, Kim YK: Transient hyperperfusion after superficial temporal artery/ middle cerebral artery bypass surgery as a possible cause of postoperative transient neurological deterioration. Cerebrovasc Dis 25:580-586, 2008

15. Komotar RJ, Starke RM, Otten ML, Merkow MB, Garrett MC,
Marshall RS, et al: The role of indirect extracranial-intracranial bypass in the treatment of symptomatic intracranial atheroocclusive disease. J Neurosurg 110:896-904, 2009

16. Liebeskind DS, Cotsonis GA, Lynn MJ, Cloft HJ, Fiorella D, Derdeyn CP, et al: Collaterals determine risk of early territorial stroke and hemorrhage in the SAMMPRIS Trial. Stroke 43:A124, 2012 (Abstract)

17. Liebeskind DS, Cotsonis GA, Saver JL, Lynn MJ, Turan TN, Cloft HJ, et al: Collaterals dramatically alter stroke risk in intracranial atherosclerosis. Ann Neurol 69:963-974, 2011

18. Perren F, Horn P, Vajkoczy P, Schmiedek P, Meairs S: Power Doppler imaging in detection of surgically induced indirect neoangiogenesis in adult moyamoya disease. J Neurosurg 103:869-872, 2005

19. Powers WJ, Clarke WR, Grubb RL Jr, Videen TO, Adams HP Jr, Derdeyn CP: Extracranial-intracranial bypass surgery for stroke prevention in hemodynamic cerebral ischemia: the Carotid Occlusion Surgery Study randomized trial. JAMA 306:1983-1992, 2011 (Erratum in JAMA 306:2672, 2011)

20. Reis CV, Safavi-Abbasi S, Zabramski JM, Gusmão SN, Spetzler RF, Preul MC: The history of neurosurgical procedures for moyamoya disease. Neurosurg Focus 20(6):E7, 2006

21. Sacco RL, Kargman DE, Gu Q, Zamanillo MC: Race-ethnicity and determinants of intracranial atherosclerotic cerebral infarction. The Northern Manhattan Stroke Study. Stroke 26:14-20, 1995

22. Siddiq F, Chaudhry SA, Khatri R, Rodriguez GJ, Tummala $\mathrm{R}$, Suri MF, et al: Rate of postprocedural stroke and death in SAMMPRIS trial-eligible patients treated with intracranial angioplasty and/or stent placement in practice. Neurosurgery 71:68-73, 2012

23. Williams JE, Chimowitz MI, Cotsonis GA, Lynn MJ, Waddy SP: Gender differences in outcomes among patients with symptomatic intracranial arterial stenosis. Stroke 38:20552062, 2007

24. Wityk RJ, Lehman D, Klag M, Coresh J, Ahn H, Litt B: Race and sex differences in the distribution of cerebral atherosclerosis. Stroke 27:1974-1980, 1996

25. Wong LK: Global burden of intracranial atherosclerosis. Int J Stroke 1:158-159, 2006

\section{Author Contributions}

Conception and design: Gonzalez, Dusick, Martin, Van de Wiele, Liebeskind, Saver. Acquisition of data: Dusick, Connolly, Bounni. Analysis and interpretation of data: Gonzalez, Dusick, Connolly, Bounni. Drafting the article: Gonzalez, Dusick. Critically revising the article: Gonzalez, Dusick. Reviewed submitted version of manuscript: Gonzalez, Dusick. Approved the final version of the manuscript on behalf of all authors: Gonzalez. Statistical analysis: Gonzalez. Administrative/technical/material support: Gonzalez, Martin. Study supervision: Gonzalez, Saver. Treating surgeon: Gonzalez, Martin. Treating surgeon, development of EDAS technical details: Gonzalez, Martin. Treating anesthesiologist, development of anesthetic guidelines for EDAS: Van de Wiele.

\section{Supplemental Information \\ Online-Only Content}

Supplemental material is available with the online version of the article.

Supplementary Materials. http://thejns.org/doi/ suppl/10.3171/2014.10.JNS141426.

\section{Correspondence}

Nestor R. Gonzalez, Department of Neurosurgery, David Geffen School of Medicine at UCLA, 100 UCLA Medical Plaza, Ste. 219, Los Angeles, CA 90095. email: ngonzalez@mednet.ucla.edu. 